\title{
Abstract and Current Literature
}

\section{Correlation of hypertension with renal function in children with obstructive uropathy}

Md. Habibur Rahman

Pediatric Nephrology, Bangabandhu Sheikh Mujib Medical University, Dhaka, Bangladesh

Objective: The aim and objective of the study was to see the correlation of hypertension with renal function and renal function with obstructive uropathy.

Methods: This prospective study was conducted in the department of Paediatric Nephrology, Bangabandhu Sheikh Mujib Medical University, Dhaka, Bangladesh over a period of two year from 01.01.2009 to 31.12.2010.In this study 41 different types of obstructive uropathy patients with age range from 4 months to 14 years (Mean age $5.7+/$ - 2.3 years) were enrolled. Patients were diagnosed by detailed history, physical examination and relevant imaging studies like ultrasound of the kidney, ureter, bladder region with post voidal residue, micturatingcystourethrogram, DMSA and DTPA renogram with total and spilt renal function. Chronic kidney disease (CKD) Stagings were done by Schwarts formula.

Results: Important observation of this study were out of 41 patients 11 (26.88\%) had hypertension 30 (73.17\%) were Normotensive. Among the hypertensive patients $5(45.45 \%)$ were in stage $\mathrm{V}$ (mean Ccr 10.7 $+/-2.57 \mathrm{ml} / 1.73 \mathrm{~m} 2 / \mathrm{min}$ ) and 2 (18.18\%) were in stage IV (Mean Ccr $22.4+/-7.58 \mathrm{ml} / 1.73 \mathrm{~m} 2 / \mathrm{min}$ ) CKD, which is statistically significant $(P<0.001)$. When normotensive patients were considered 13 (40.62\%), $5(15.62 \%)$ and $4(13.33 \%)$ were in stage III, IV, and V CKD respectively. On the other hand, when CKD status were correlated with types of obstructive uropathy posterior urethral valve 25 (61\%)were present in the majority cases and all $(100 \%)$ of the patients had different grades of CKD.

Conclusion: It was observed from this study, patients with CKD due to obstructive uropathy may be Normotensive ever in stage $\vee C K D$, and posterior urethral valve was the commonest cases of obstructive uropathy in children.

PediatrNephrol (2013) 28:1379-1532
Congenital Acute Lymphoblastic Leukemia: A Rare Presentation in a One Month Old Boy

Hafiz MG*and Khaleque MA

A one month old boy of non-consanguineous parents was admitted with gradual distension of abdomen, yellow coloration of whole body, progressive pallor and respiratory distress since birth. Septic investigations were done and found negative. His complete blood count with peripheral blood film examination revealed hyperleukocytosis, thrombocytopenia and presence of significant number of blasts cell. Cerebrospinal fluid (CSF) examination showed central nervous system (CNS) status $3(\mathrm{e}-5 \mathrm{WBC} / \mathrm{cmm} 3$ with blasts). Other causes of leukemoid reaction were ruled out. Karyotyping had done and found normal chromosomal pattern (46XY). Bone marrow aspiration findings were suggestive of ALL-L1.His myeloperoxidase (MPO) and Sudan black stain was negative but Periodic acid Schiff (PAS) stain was positive. Immunophenotype showed blasts cells which were positive for CD5(1.10\%), CD8(0.4\%), CD10(0.74\%), CD13(13.7\%) and CD19(62.4\%). Finally, the boy was diagnosed as congenital acute lymphoblastic leukemia (ALL-L1, Blineage, CNS status 3). Following chemotherapy, the child suddenly deteriorated and on the second day of therapy suddenly he expired possibly due to leukostasis or coagulopathy or non-responsive of drugs or MLL gene translocation. So, congenital acute lymphoblastic leukemia (CALL) should be kept in mind in a newborn child with clinical features of sepsis, leukocytosis, thrombocytopenia, huge hepatosplenomegaly. Journal of Pediatrics and Therapeutics, Volume 4, Issue 2, Pages 1-4

Received: January24, 2014; Accepted April 04, 2014; Published April 07, 2014(Online)

Impact of Study Oximeter Masking Algorithm on Titration of Oxygen Therapy in the Canadian Oxygen Trial

Barbara Schmidt, Robin S. Roberts, Robin K. Whyte, Elizabeth V. Asztalos, Christian Poets, Yacov Rabi, et al.

Objective: To compare oxygen saturations as displayed to caregivers on offset pulse oximeters in the 2 groups of the Canadian Oxygen Trial. 
Study design: In 5 double-blind randomized trials of oxygen saturation targeting, displayed saturations between $88 \%$ and $92 \%$ were offset by $3 \%$ above or below the true values but returned to true values below $84 \%$ and above $96 \%$. During the transition, displayed values remained static at $96 \%$ in the lower and at $84 \%$ in the higher target group during a $3 \%$ change in true saturations. In contrast, displayed values changed rapidly from $88 \%$ to $84 \%$ in the lower and from $92 \%$ to $96 \%$ in the higher target group during a 1\% change in true saturations. We plotted the distributions of median displayed saturations on days with $>12$ hours of supplemental oxygen in 1075 Canadian Oxygen Trial participants to reconstruct what caregivers observed at the bedside.

Results: The oximeter masking algorithm was associated with an increase in both stability and instability of displayed saturations that occurred during the transition between offset and true displayed values at opposite ends of the 2 target ranges. Caregivers maintained saturations at lower displayed values in the higher than in the lower target group. This differential management reduced the separation between the median true saturations in the 2 groups by approximately $3.5 \%$.

Conclusions: The design of the oximeter masking algorithm may have contributed to the smaller-thanexpected separation between true saturations in the 2 study groups of recent saturation targeting trials in extremely preterm infants.

The Journal of Pediatrics, Volume 165, Issue 4 , Pages 666-671. Received 20 February 2014; accepted 13 May 2014. published online 26 June 2014.

\section{Trends in Pharmacotherapy for Neonatal Hypotension}

Danielle R. Rios, Brady S. Moffett, Jeffrey R. Kaiser,

Received 13 February 2014; received in revised form 20 May 2014; accepted 3 June 2014. published online 18 July 2014.

Objective: To determine trends in pharmacotherapy for neonatal hypotension in all infants and in extremely low birth weight (ELBW, birth weight 300-1000 g) infants.

Study design: We queried the Pediatric Health Information System database for all infants d"28 days with a diagnosis code for hypotension that were discharged between January 2001 and December 2012. Patients were excluded if they had complex congenital heart disease or cardiac surgery, sepsis or meningitis, or had extracorporeal membrane oxygenation. We determined trends in pharmacotherapy for hypotension in all infants and ELBW infants, an especially vulnerable group.

Results: A total of 8019 hypotensive infants met study criteria. The 2 most prescribed medications were dopamine (65.3\%) and dobutamine (19.9\%). For 1487 hypotensive ELBW infants, the 2 most prescribed medications were dopamine (83.4\%) and hydrocortisone (33\%). During the study period, the use of dobutamine decreased, and hydrocortisone and vasopressin use increased for all infants and for ELBW infants.

Conclusions: Treatment of neonatal hypotension varies widely between institutions and individual practitioners, and pharmacotherapy for neonatal hypotension has changed over the past decade. Although dopamine and dobutamine were the most frequently used agents, their use has declined and the uses of hydrocortisone and vasopressin have increased.

The Journal of Pediatrics Volume 165, Issue 4 , Pages 697-701.e1, October 2014 